This item was submitted to Loughborough's Research Repository by the author.

Items in Figshare are protected by copyright, with all rights reserved, unless otherwise indicated.

\title{
An evaluation of sit to stand devices for use in rehabilitation
}

\section{PLEASE CITE THE PUBLISHED VERSION}

https://doi.org/10.1007/978-3-319-96071-5_81

\section{PUBLISHER}

(C) Springer

\section{VERSION}

AM (Accepted Manuscript)

\section{PUBLISHER STATEMENT}

This is a pre-copyedited version of a contribution published in Bagnara, S. ... et al. (eds.) 20th International Ergonomics Association (IEA2018): Volume VII: Ergonomics in Design, Design for All, Activity Theories for Work Analysis and Design, Affective Design published by Springer. The definitive authenticated version is available online via https://doi.org/10.1007/978-3-319-96071-5_81

\section{LICENCE}

CC BY-NC-ND 4.0

\section{REPOSITORY RECORD}

Fray, Mike, Sue Hignett, A. Reece, S. Ali, and L. Ingram. 2019. "An Evaluation of Sit to Stand Devices for Use in Rehabilitation”. figshare. https://hdl.handle.net/2134/35062. 


\section{An evaluation of sit to stand devices for use in}

\section{rehabilitation}

M. Fray ${ }^{1}$, S. Hignett ${ }^{1}$, A. Reece ${ }^{1}$, S. Ali ${ }^{1}$, L. Ingram ${ }^{1}$

${ }^{1}$ Loughborough Design School, Loughborough University, UK. M.J.Fray@lboro.ac.uk

Abstract.

There are many assistive devices to help with raising a person from a seat. These devices are considered active as they require some balance, trunk control and weightbearing ability. There is concern that this movement is mostly passive due to fixation at the trunk and knee. This study explores the movement patterns in sit to stand tansfers active and assisted.

Study Design: A fully squared repeated measures design was use. All participants $(n=20)$ used all conditions $(n=7)$ in a balanced order. Transfers were recorded with; video recordings, a 6 dimensional force plate, hip, knee and ankle positions were recorded with motion capture. Subjective evaluations for comfort and security were completed. Physical data was compared with ANOVA calculations with Bonferroni corrections.

Results: Device G scored highest for comfort, knee support and overall preference. Sling movement had a negative effect on the sensations of comfort and security. The motion analysis of the flexible knee support showed:

- People push into the floor and CoP moved towards the toe

- More anterior knee movement $(\mathrm{P}<0.05)$.

- More bodyweight through feet $(\mathrm{P}<0.05)$.

- Quicker transfer of weight onto feet

- Very low bodyweight was recorded in all lowering actions.

The use of a flexible knee support raised the subjective and physical performance of the assistive device and may improve rehabilitation repsonses.

Keywords: Patient transfers, rehabilitation, assistive technology. 


\section{Introduction}

When a patient requires an assistive device to help with the activity of raising from a seat there are many devices that can deliver the support and movement needed. The functional movement for transport, patient movement or hygiene requirements is common place in care settings. The design and action of most sit to stand assistive devices is considered to be active as the device requires some balance, trunk control and weight-bearing ability in the person being assisted. There is concern that the movement with current devices is mostly passive and the support and structure of the device creates and controls all movements in the lower limbs due to fixation at the trunk and knee This study explores the patterns of movement in natural movement against the movements seen in assisted transfers with a range of devices.

Sit to stand (STS) is a function performed regularly by ambulatory adults to function independently and maintain activities of daily living (ADL's). The primary goal of the STS task is to move the total body centre of mass (CoM) from a relatively low, fully supported position to an upright, stable, standing position (Mathiyakom, 2005). The inability to rise from a sitting position is recognised by the World Health Organization (WHO, 1980) as a disabling condition. Many studies have looked the effects of age, osteoarthritis, musculoskeletal pain, joint arthroplasty, cerebrovascular incidents and neurological conditions on a person's ability to perform the STS movement effectively (Burnfield, 2013; Rutherford et al, 2014; Sibella, 2003; Munton et al, 1984; Pai, 1991; Hughes and Schenckman, 1996; Coghlin and McFadyen, 1994, Schenkman et al, 1996).

A significant number of injuries result from transferring patients (Stubbs, 1986). The implementation of SPH techniques and devices has helped reduce work-related injuries etc (Slusser et al, 2012, Sparkes, 2000). Sit to stand devices are used as a rehabilitation tool. Concerns regarding the effectiveness of STS devices suggest they discourage active muscle engagement. Campo et al, (2013) examined the effects on patient mobility with the use of SPH devices or not and showed similar mobility outcomes in both groups. Studies have looked at the kinematics of device assisted transfers in adults with no disability and those that suffered a stroke (Burnfield, 2013 and 2012). Other studies report reduction in work related musculoskeletal injuries in healthcare professionals (Ruszala and Musa, 2004, Garg and Kapellusch, 2012). There is little evidence to guide decision making for the use of STS devices (Campo, 2013, Burnfield et al , 2012, Arnold, 2013). To date, only one study compared joint motion and CoP in device-assisted sit-to-stand transfers (Jeysurya (2013).

\section{Methods}

\section{$2.1 \quad$ Objectives}


To explore the relationships between the movement patterns in an unassisted active sit to stand, and active stand to sit, and the movements recorded when using sit to stand assistive devices for both tasks. This study compares all aspects of human movement and subjective assessment in the STS transfers. The trial followed a fully squared repeated measures design where all participants $(n=20)$ were exposed to all conditions ( $n=7$, unassisted active plus 6 devices). The order of presentation was balanced to give appropriate comparison. Participants were required to complete 3 sit to stands and 3 stand to sits with each device. All data collection methods were piloted with a small number of participants before committing to the full trial.

The study compared an unassisted action with the device assisted transfers. The active movement was self-selected. 5 comparison devices were purchased (B-F) from the marketplace and adapted to allow for data collection. Device $G$ was a prototype device which had an innovative flexible silicone knee support. The sling fitting, set up and controls were controlled by the experimenter. The conditions were:
A. Active motion no assistance (Control)
B. Knee/shin support, lever lift, sling, flat footplate
C. Knee/shin support, lever lift adjustable, sling, flat footplate
D. Knee support, Straight line lift, sling, flat footplate
E. Knee support, lever lift, sling, flat footplate
F. Knee/shin support, lever lift, sling, angled footplate
G. Silicone knee support, lever lift, sling, flat footplate

Participants. Participants were acquired from local recruitment. All were required to be 55+ years, no restrictions were placed medical history but each had to be able to complete the sit to stand action independently. For all transfers the participants were requested to raise/lower themselves to the highest/lowest position possible with the device.

\subsection{Subjective Data Collection}

After each set of transfers with any device ( $\mathrm{n}=3$ repetitions) a range of subjective evaluations were requested from the participant inquiring: Comfort; Security; Sling fitting; knee support; Overall performance. All subective evaluations were recorded on 5 point likert scales ( 5 being preferred score). After all devices were completed a post study interview was conducted. The experimenter recorded verbal comments and the particiant was asked to rate their preference or dislike for various aspects of the devices.

- Overall preference of device

- Preferred slings

- Preferred knee support

\subsection{Physical Data Collection}

The movements completed were recorded with 3 methods; video recordings were taken as a record of each transfer, the ground reaction force was recorded using a 6 dimensional force plate, the limb positions and limb angles were recorded with motion capture (Codamotion with Odin Software). The data used for analysis was: 
- Force measured through force plate

- Centre of Pressure location (anterior to posterior, AP)

- Joint positions from motion capture

o $\quad$ Foot - Toe, 5th Metatarsal and Heel; Fibula - Lateral Malleolus and Fibula Head; Femur - Epicondyle and Greater Trochanter; Pelvis - Anterior and Posterior Iliac Spine, Shoulder - Acromion

o Joint Angles 3D Eular angle calculations for ankle, knee, hip

- Video recordings were used for clarification.

\subsection{Analysis and Presentation of Data}

The physical data was filtered for missing and erroneous data. The movement started when the hip marker was raised/lowered $20 \mathrm{~mm}$ and finished when the hip marker ceased movement. For comparison between transfers data were normalized to percentage figures e.g. time, weight, centre of pressure across the foot length etc. After all devices a post study interview was conducted reviewing preferences. Physical data was compared with ANOVA calculations with Bonferroni corrections.

\section{$3 \quad$ Results}

Participant characteristics $(\mathrm{n}=20)$ were all over 55 years, mass 44.4-109.9 kgs, height $147.3-187.9 \mathrm{~cm}$. Medical histories were recorded but none were excluded. The physical data was filtered for missing data and erroneous data which was all removed. The movement was identified as starting at when the hip marker was raised/lowered $20 \mathrm{~mm}$ and finished when the hip marker ceased movement. This gave comparable time frames with only the movement phases being included. To allow comparison between transfers many data were normalised to percentage figures e.g. time, weight, centre of pressure across the foot length etc.

\subsection{Subjective Results}

The subjective scores were recorded on 5 point Likert scales. Judgements for raising and lowering were combined. 5 being the positive score and 1 being the least regarded. Graph 1 showed that for comfort the flexible support $G$ and Device $C$ were the most comfortable. Graph 1 Average comfort during the movement 


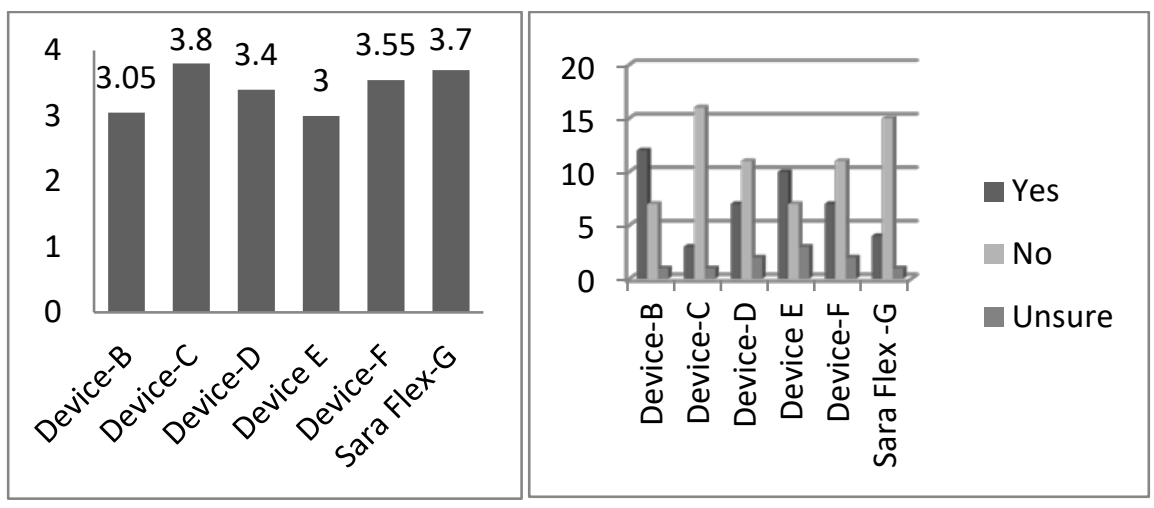

Graph 1. Comfort Scores.

Graph 2. Sling Movement.

The sling movement had a clear relationship with the sensations of comfort and security. Graph 2 showed the frequency that reported the sling moving. Devices B, E, $\mathrm{D}$ and $\mathrm{F}$ reported the most movement with $\mathrm{C}$ and $\mathrm{G}$ being the least movement. Graph 2. Participants reporting sling movement

After the participant had used all devices for all repetitions the post-trial interview revealed a preference for the flexible device $(G)$. Participants reported the most preferred and the least preferred across the devices. Graph 3 indicates the most preferred votes for the flexible support $G$ however feedback recorded that some thought the flexibility felt less secure than others in the range.

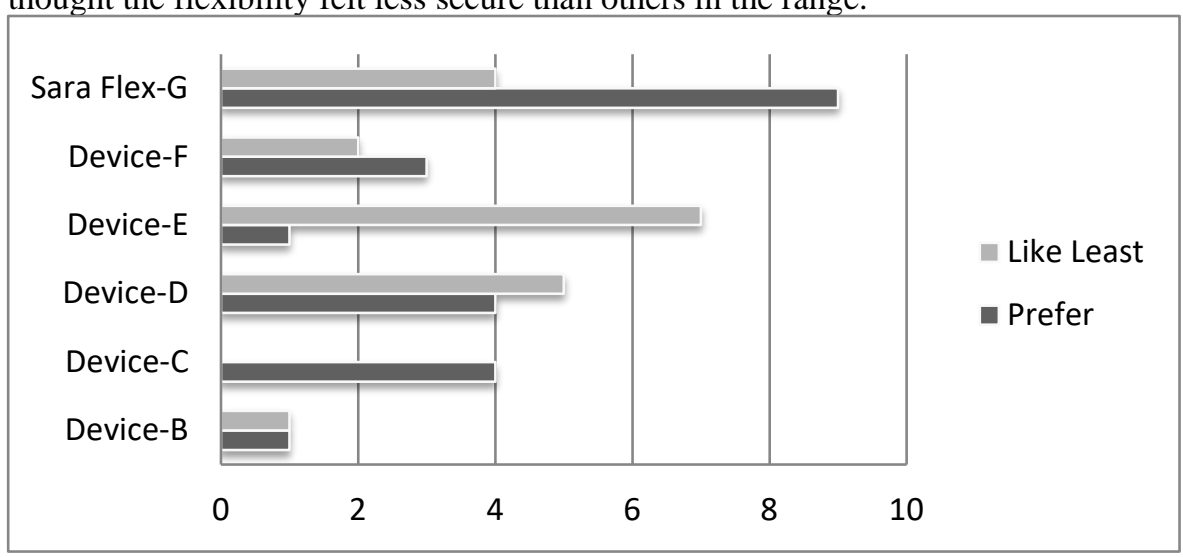

Graph 3 Preference and dislike ratings for devices overall

Graph 4 shows the ratings for the sling preference. Devices B and E recorded more negative views than positive. Specifically E had a slow and very vertical lift pattern which increased the slippage of the sling against the participant. The devices D, C and $\mathrm{G}$ had more positive than negative ratings. The flexible knee device showed the most positive response. Interestingly $\mathrm{F}$ and $\mathrm{G}$ had identical slings but the combination with the silicone knee support affected the ratings. 


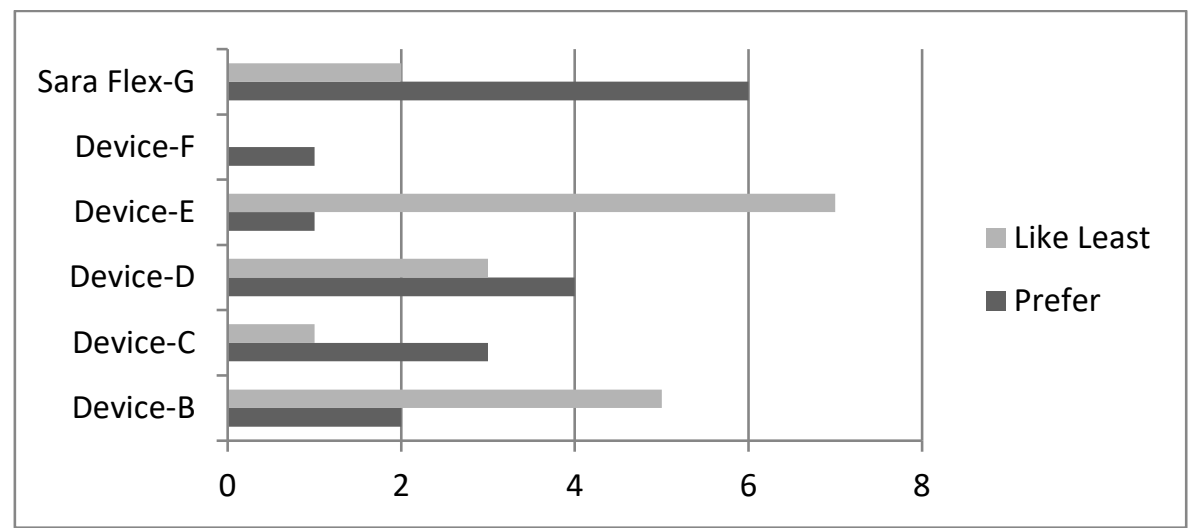

Graph 4 Preference and dislike ratings for slings

Whilst some participants considered the silicone knee support to lack security Graph 5 show the overwhelming preference for the flexible knee support (G) during the trial.

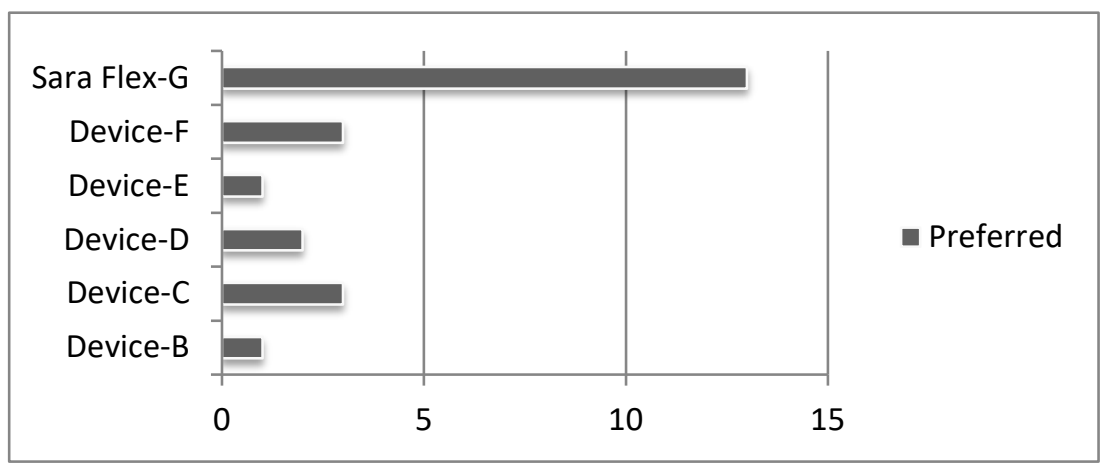

Graph 5. Preference for knee supports

\subsection{Physical Results}

The first results were used to understand the movement components of the active sit to stand. There was good variation between participants with good correlation between the repetitions for each participant. Graph 6 shows the \% bodyweight (\%BW) and the position of the centre of pressure (CoP). The CoP plot shows how the weight starts approx. $60 \%$ towards the heel and as the person stands the weight moves forward towards the toe and then back again once in standing. The \%BW is characterised by a rapid onset of force as the weight comes off the seat and there is maximum acceleration up to standing. 


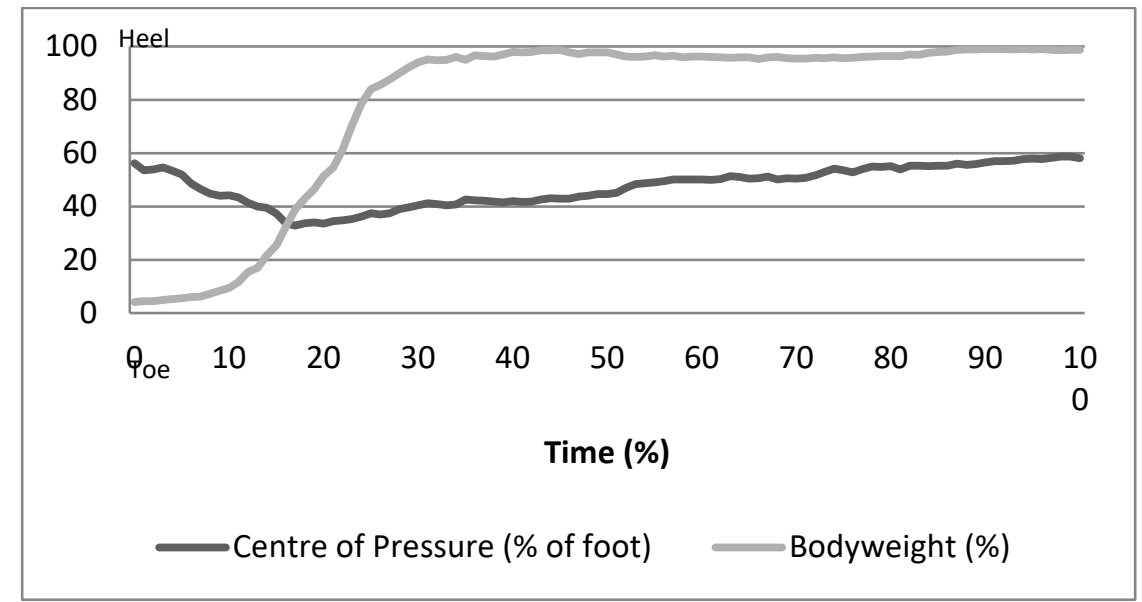

Graph 6 Active Sit to Stand - Centre of Pressure and \% Bodyweight

The main aim of this study was to explore the relationship between the movement pattern in the active tasks and those reported in the assisted transfers. The analysis of the movement for the active sit to stand showed forward movement of the knee with forward lean of the trunk to allow the weight to be taken on the feet to allow vertical acceleration into standing and a corresponding extension at hip, knee and ankle. The following graphs show some movement comparisons between the devices and the active sit to stand.

Graph 7 shows the horizontal movement of the knee as it comes forward over the toes in standing. The closest device to the active curve is the flexible knee support which is significantly higher movement than all other devices (ANOVA, $\mathrm{P}<0.05$ and 50\% and $99 \%$ of transfer).

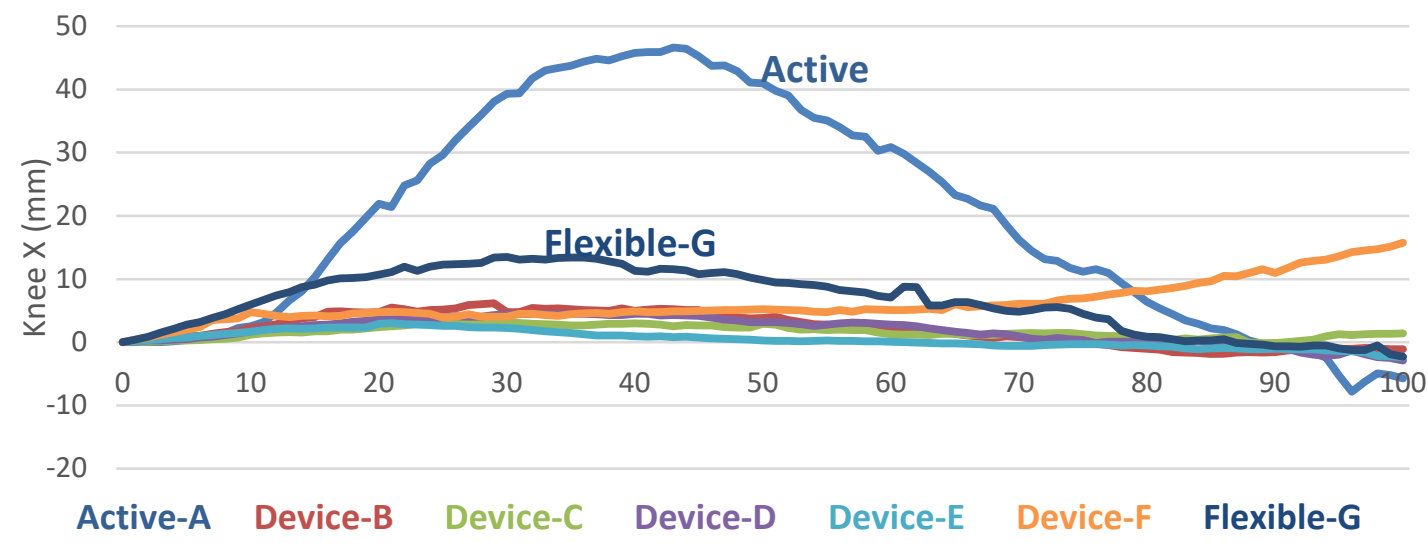

Graph 7. Forward Knee Movement 
As the person moves forward the angle closes and the curve of the median hip angle drops below the assisted movement curves. The backward lean into the sling allows only the steady increase from $90^{\circ}$ to upright approximately $180^{\circ}$ for all devices.

Similar comparisons were seen in the angles of knee and ankle where movement was seen in the active but much less in the device assisted. The clearest benefit of the flexible knee support was the effect on the ground reaction force Graph 8. Device G allowed up to $70 \%$ of the body weight to be measured at the standing position which was significantly higher than most other devices $(\mathrm{p}<0.05)$.

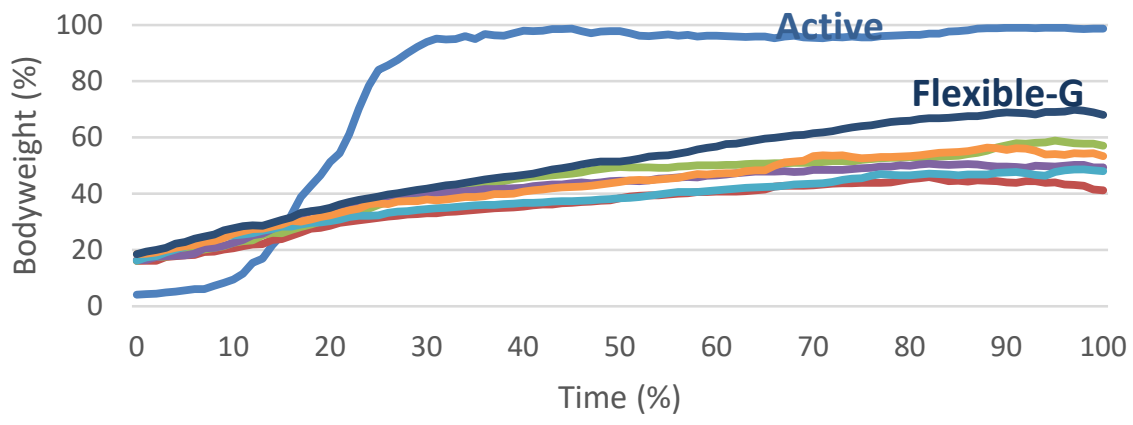

\section{Active-A Device-B Device-C Device-D Device-E Device-F} Flexible-G

Graph 8. \% Bodyweight for the Sit to Stand Action

The experimental team expected the CoP for the devices to be much further to the heel as the centre of gravity and centre of mass is supported by the device outside the base of support. The results show that the CoP movement is much closer to the active pattern than expected. This is explained by the response to being pulled forward by the sling. The participant braced themselves against the footplate and hence the CoP moved forward towards the toe. The data for the stand to sit actions the patterns of movement for horizontal knee movement, hip, knee and ankle angles were all very similar and did not follow the active movement pattern. There was however a significant reduction in the bodyweight data which was very small for all assisted sitting actions. This large reduction in ground reaction force is a key finding. The video analysis showed that the participants lowered themselves into the knee support and took tension in the sling as per the instruction. This created a physical support between the two fixed positions and made the knee the primary weight bearing structure and not the feet. 


\section{$4 \quad$ Concluding Remarks}

The innovative device G showed some positive differences to the other devices in this trial and in several data sets was closest to the active pattern of movement. This investigation reported:

- When assisted people push into the floor and CoP moved towards the toe

- When over lifted CoP moved towards the toe as weight decreases

- Allowed more forward movement at the knee

- Allowed more weight through feet compared to other devices

- Showed quicker transfer of weight onto feet like 'normal' stand

- During lowering (stand to sit) very low bodyweight was recorded from all devices.

The distribution of the bodyweight in the different movements is an interesting finding when considering the question of using the devices as an aid to rehabilitation. The flexible knee support allowed more forward knee movement, allowed more bodyweight through the feet and gave one of the closest $\mathrm{CoP}$ movement patterns to the active unassisted transfer. With these differences the device with the flexible knee support was still the most preferred device in this trial. These component actions show that this device encouraged the participant to be more active through sitting and standing assisted transfers.

Specifically, it should be noted that for an unassisted active stand to sit transfer stronger eccentric muscle activation is required to support lowering. Adding a level of voluntary control in the persons own muscles to support their descent would raise the activity level for people who are assisted. This increase in activity will improve their route to improved function and rehabilitation goals. In our modern healthcare systems this may support an earlier access to rehabilitation and lead to an earlier recovery.

\section{References}

Burnfield JM, McCrory B. (2013). Comparative kinematic and electromyographic assessment of clinician- and device-assisted sit-to-stand transfers in patients with stroke. Phys Ther.

Burnfield JM, Shu Y, Buster TW, et al. (2012). Kinematic and electromyographic analyses of normal and device-assisted sit-to-stand transfers. Gait Posture. 2012;36:516-522

Campo M, Shiyko MP, Margulis H, Darragh AR. (2013). The effect of a safe patient handling program on rehabilitation outcomes. Arch Phys Med Rehabil. 2013;94:17-22.

Carr JH, Gentile AM. The effect of arm movement on the biomechanics of standing up. Hum Mov Sci. 1994;13:175-193

Coghlin SS, McFadyen BJ (1994). Transfer strategies used to rise from a chair in normal and low back pain subjects. Clin Biomech. 1994;9:85-92

Hughes MA, Schenkman ML. (1996). Chair rise strategy in the functionally impaired elderly. J Rehabil Res Dev. 1996;33:409-412.

International Classification of Impairments, Disabilities and Handicaps. Geneva, Switzerland: World Health Organization,1980 
Janssen W. Bussmann H. Henk S. (2002). Determinants of the sit to stand movement. Journal of American Physical Therapy Association. Phys Ther. 2002; 82: 866-897

Jeysurya J. Van der Loos M. (2013). Comparison of seat, waist, and arm sit-to-stand assistance modalities in elderly population. University of British Columbia. 2013; 50: 835-844

Mathiyakom, K. McNitt Grey, J.L. (2005). Modifying center of mass trajectory during sitto-stand tasks redistributes the mechanical demand across the lower extremity joints. Clinical Biomechanics.

Munton JS, Ellis MI, Wright V. (1984). Use of electromyography to study leg muscle activity in patients with arthritis and in normal subjects during rising from a chair. Ann Rheum Dis. 1984;43:63-65.

Pai YC, Rogers MW. Speed variation and resultant joint torques during sit-to-stand. Arch Phys Med Rehabil. 1991;72:881-885.

Rutherford D.J, Hurley S.T, Hubley-Kozey C. (2014). Sit-to-stand transfer mechanics in healthy older adults: a comprehensive investigation of a portable lifting-seat device. Disabil Rehabil Assist Technol.

Ruszala, S. Musa, S. (2005). An evaluation of equipment to assist patient sit-to-stand activities in physiotherapy. Physiotherapy. 2005;91:35-41

Sibella F. Galli, M. (2003), Biomechanical analysis od sit to stand movement in normal and obese subjects. Clin Biomech. 2003; 18:745-750

Schoen DC. Low back pain. Orthopaedic Nursing. 2004;23:154-157.

Schenkman ML, Riley PO, Pieper C. (1996). Sit to stand from progressively lower seat heights: alterations in angular velocity. Clin Biomech. 1996; 11:153-158.

Slusser LR, Rice MS, Miller BK. Safe patient handling curriculum in occupational therapy and occupational therapy assistant programs: a descriptive study of school curriculum within the United States of America. Work. 2012;42:385-392

Sparkes V. Physiotherapy for stroke rehabilitation: a need for evidence-based handling techniques: literature review. Physiotherapy. 2000;86:348-356.

Stubbs DA, Buckle PW, Hudson MP, et al. Backing out: nurse wastage associated with back pain. Int J Nurs Stud. 1986;23: 325-336. 